\title{
Atuação da fisioterapia aquática em pacientes com lombalgia: uma revisão
}

\section{sistemática}

\author{
Performance of aquatic physiotherapy in patients with low back given: a systematic review \\ Realización de fisioterapia acuática em pacientes con espalda baja: una revisión sistemática
}

Recebido: 24/10/2021 | Revisado: 27/10/2021 | Aceito: 27/10/2021 | Publicado: 30/10/2021

\author{
Brenda Karoline Teles Araújo \\ ORCID: https://orcid.org/0000-0002-8737-8537 \\ Centro Universitário Santo Agostinho, Brasil \\ E-mail: brendakarolinet@live.com \\ Luana Janaína da Silva Vasconcelos \\ ORCID: https://orcid.org/0000-0003-2109-9161 \\ Centro Universitário Santo Agostinho, Brasil \\ E-mail: luanna_vasconcelos17@hotmail.com \\ Jordano Leite Cavalcante de Macêdo \\ ORCID: https://orcid.org/0000-0003-0283-9005 \\ Centro Universitário Santo Agostinho, Brasil \\ E-mail: jordano_cm@yahoo.com.br
}

\begin{abstract}
Resumo
A lombalgia ou dor lombar é uma disfunção que pode ser de origem traumática, biomecânica ou biopsicossocial, que acomete ambos os sexos e que pode ser caracterizada pela presença de dor de caráter agudo ou crônico. O estudo objetivou buscar na literatura evidências sobre atuação e efetividade da hidroterapia em pacientes com lombalgia. Foi realizado um estudo de revisão sistemática nas bases de dados: PEDro, Scielo, PubMed usando os descritores em espanhol, inglês e português publicados nos últimos anos. Após a análise criteriosa dos resultados obtidos, foram selecionados 04 artigos que contribuem para a realização do estudo. Destaca-se que os autores mostram que a hidroterapia é eficaz no tratamento da dor e melhora a qualidade de vida dos indivíduos, é importante citar a necessidade de mais ensaios clínicos na área que mostrem a evidência da hidroterapia em pacientes com lombalgia.

Palavras-chave: Dor lombar; Exercícios aquáticos; Hidroterapia.
\end{abstract}

\begin{abstract}
Low back pain or low back pain is a disorder that can be of traumatic, biomechanical or biopsychosocial origin, affecting both sexes and that can be characterized by the presence of pain of an acute or chronic nature. The study aimed to search the literature for evidence on the performance and effectiveness of hydrotherapy in patients with low back pain. A systematic review study was carried out in the following databases: PEDro, Scielo, PubMed using the descriptors in Spanish, English and Portuguese published in recent years. After careful analysis of the results obtained, 04 articles that contribute to the study were selected. It is noteworthy that the authors show that hydrotherapy is effective in the treatment of pain and improves the quality of life of individuals, it is important to mention the need for more clinical trials in the area to show evidence of hydrotherapy in patients with low back pain.
\end{abstract}

Keywords: Low back pain; Water exercises; Hydrotherapy.

\section{Resumen}

La lumbalgia o lumbalgia es un trastorno que puede ser de origen traumático, biomecánico o biopsicosocial, afectando a ambos sexos y que puede caracterizarse por la presencia de dolor de carácter agudo o crónico. El estudio tuvo como objetivo buscar en la literatura evidencia sobre el rendimiento y la efectividad de la hidroterapia en pacientes con dolor lumbar. Se realizó un estudio de revisión sistemática en las siguientes bases de datos: PEDro, Scielo, PubMed utilizando los descriptores en español, inglés y portugués publicados en los últimos años. Tras un cuidadoso análisis de los resultados obtenidos, se seleccionaron 04 artículos que aportan al estudio. Es de destacar que los autores demuestran que la hidroterapia es efectiva en el tratamiento del dolor y mejora la calidad de vida de los individuos, es importante mencionar la necesidad de más ensayos clínicos en el área para mostrar evidencia de hidroterapia en pacientes con lombalgia.

Palabras clave: Lumbalgia; Ejercicios acuáticos; Hidroterapia. 


\section{Introdução}

A coluna lombar consiste numa estrutura móvel do esqueleto axial, que geralmente sofre em demasia com a ação do tempo. Isso ocorre tanto aos esforços dinâmicos relacionados a deslocamentos, transporte de cargas e a utilização de escadas, quanto aos esforços estáticos relacionados com a sustentação de objetos pesados e com a adoção de posturas incorretas, com a restrição de movimentos, contribuindo para a ocorrência de lesões musculares (Almeida, et al., 2019).

A lombalgia ou dor lombar é uma disfunção que pode ser de origem traumática, biomecânica ou biopsicossocial, que acomete ambos os sexos e que pode ser caracterizada pela presença de dor de caráter agudo ou crônico (Amorim et al., 2019). Sua prevalência tem sido estimada em vários estudos representando diferentes populações. A prevalência ao longo da vida da lombalgia não específica é estimada em mais de $70 \%$ nos países industrializados (com uma prevalência em um ano entre 15 e $45 \%$, e incidência em adultos de 5\% por ano). A taxa de prevalência nas crianças e adolescentes aproxima-se da dos adultos, aumentando e atingindo o pico entre os 35 e os 55 anos (Meirinho, et al., 2018).

Segundo (Monteiro, 2019) a Fisioterapia Aquática ou Hidroterapia é a especialidade da Fisioterapia que usa a o ambiente aquático, a piscina terapêutica, aquecida e com acessibilidade, para tratar das diversas áreas e níveis de saúde, utilizando a mecânica dos movimentos e dos fluidos; efeitos fisiológicos e terapêuticos; equipamentos; além de métodos e técnicas específicos.

A Fisioterapia Aquática tem uma série de benefícios quando comparada com exercícios em solo. A temperatura elevada de piscinas de hidroterapia pode também diminuir a dor e a rigidez como promover o relaxamento (Hinman, et al., 2007). A flutuabilidade reduz a quantidade de carga que passa por uma articulação, o que permite aos pacientes executarem uma cadeia fechada funcional e exercícios que podem não ser possíveis em solo (Bartels, et al., 2009).

A execução dos movimentos, com o corpo submerso na água, reduz o estresse articular, diminuindo o impacto sob as articulações e o risco de lesões, ajuda a aumentar a amplitude dos movimentos sem a resistência do atrito, auxiliando a movimentação (Saltiél, et al., 2018).

Segundo (Gonçalves \& Vilarta, 2004), a qualidade de vida ligada à saúde consiste em se sentir bem, na ausência de doenças significativas, que poderão se tornar impedimento para a realização de habilidades e capacidades; compreende à vontade para envolver-se com os múltiplos aspectos e objetos que a convivência, o trabalho e o lazer podem proporcionar à vida plena de valores e possibilidades.

Este estudo tem como objetivo descrever os efeitos fisiológicos da imersão em água aquecida para o tratamento da lombalgia, identificando as principais técnicas de reabilitação em ambiente aquático para o tratamento da dor lombar demonstrando a influência da abordagem na qualidade de vida dos pacientes.

\section{Metodologia}

Este estudo refere-se em uma revisão sistemática de literatura, de caráter qualitativo, utilizando-se a escala PEDro, desenvolvida pela Physiotherapy Evidence Database para ser empregada em estudos de intervenção e tem uma pontuação total de até 10 pontos, incluindo critérios de avaliação de validade interna e apresentação da análise estatística empregada. Visando quantificar a qualidade dos ensaios clínicos publicados, de forma a guiar os usuários sobre os aspectos meritórios de cada publicação e facilitar a identificação rápida de estudos que contenham informações suficientes para a prática profissional. Essa escala é composta por 11 critérios e a pontuação final é gerada através da somatória de dez dos onze critérios, objetivando responder a seguinte questão problema: “A fisioterapia aquática é benéfica para o tratamento da lombalgia?” e, posteriormente, montar uma tabela de resultados.

Foi avaliada a efetividade da Fisioterapia Aquática no tratamento da lombalgia, analisando através da intervenção se é eficaz para tratamento e se há uma melhora no quadro álgico, solucionando assim o problema em questão. 
Utilizou-se para a coleta a estratégia PICO, para coletar os dados e resultados da pesquisa. Nesse modelo considera-se 4 itens, P: população, paciente ou problema abordado; I: a intervenção, indicação ou interesse; C: intervenção de comparação, placebo ou não-intervenção; O: resultado esperado (OUTCOME). Foi realizado o levantamento de informações de fevereiro de 2021 até novembro de 2021, nas bases de dados da plataforma da Biblioteca Virtual em Saúde (BVS) utilizando artigos nas bases de dados: Scientific Eletronic Library Online (SCIELO), Medical Literature Analysis and Retrieval System Online (MEDLINE), PubMed e Banco de dados de evidências de fisioterapia (PEDro). Com a utilização dos descritores: Dor lombar, Exercicíos Aquáticos, Terapia Aquática e Hidroterapia e os descritores em inglês - "low back pain" or "lower back pain" and "aquatic exercices" or "aquatic therapy" or "hydrotherapy".

A pesquisa foi realizada por dois pesquisadores independentes. A seleção dos artigos foi feita através da leitura do título, depois pela leitura do resumo e, por último, a leitura completa do artigo. Como critério de inclusão foi utilizado ensaios clínicos e estudo randomizado controlado em português, espanhol e inglês, publicados em bases de dados nacionais e internacionais nos últimos anos, com score PEDro satisfatório e insatisfatório para comparação. Como critério de exclusão será optado por artigos que não apresentem o tema proposto, artigos incompletos, que não é referente à pesquisa, como por exemplo, estudo de caso. A revisão foi submetida e aprovada na plataforma PROSPERA contendo no trabalho cientifico todas as regras desde a estruturação, citação e referências.

\section{Resultados e Discussão}

$\mathrm{Na}$ análise dos resultados foram considerados todos os critérios de seleção, sendo selecionados para compor o estudo somente artigos científicos que apresentaram informações pertinentes à pesquisa, e que responderam ao objetivo do estudo (Figura 1). A pesquisa ocorreu mediante leitura completa de todos os estudos selecionados na íntegra, e foram caracterizados a partir de informações como ano de publicação, base de dados, periódico, tipo de estudo, objetivos da pesquisa, autores, título do artigo.

Figura 1 - Fluxograma de identificação e seleção de artigos. Teresina-PI, 2021.

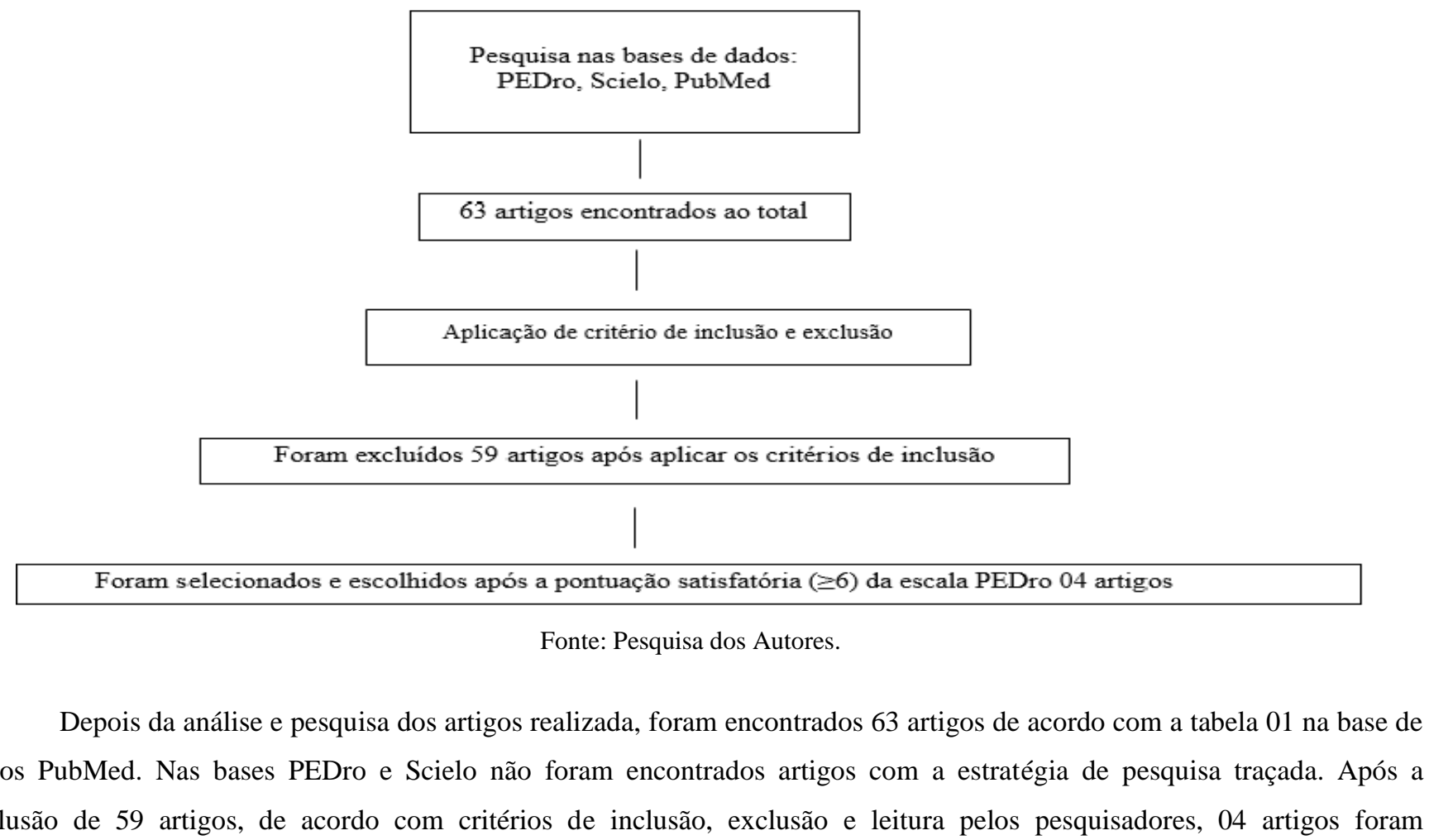


selecionados (Figura 1). Eles obtiveram nota $\geq 6$, sendo classificados como de alta qualidade. Observa-se na Tabela 2 que todos os artigos apresentaram critérios de elegibilidade, nenhum realizou alocação secreta dos sujeitos, nenhum fez o "cegamento" dos sujeitos, nenhum fez "cegamento" dos terapeutas, todos tiveram semelhança inicial entre os grupos, comparação intergrupos, medidas de precisão e variabilidade, acompanhamento adequado e distribuição aleatória. Nenhum em sua metodologia apresentou "cegamento" dos avaliadores, e todos fizeram análise da intenção de tratamento.

Tabela 1 - Bases de dados usadas para pesquisa dos artigos utilizados. Teresina-PI, 2021.

\begin{tabular}{|l|l|}
\hline Base de Dados & Número de artigos encontrados \\
\hline PEDro & 0 \\
Scielo & 0 \\
PubMed & 63 \\
\hline
\end{tabular}

Fonte: Pesquisa dos Autores.

Tabela 2 - Classificação dos ensaios clínicos de acordo com a escala PEDro. Teresina-PI, 2021.

\begin{tabular}{|c|c|c|c|c|}
\hline ESCALA PEDro & ANGIONI, et al., 2019 & ANDRADE, et al., 2017 & $\begin{array}{l}\text { CARUS, et al., } \\
007\end{array}$ & $\begin{array}{l}\text { GUSI, et al., } \\
2006\end{array}$ \\
\hline $\begin{array}{lll}\text { 1. } & \text { Critério } \\
& \text { elegibilidade }\end{array}$ & SIM & SIM & SIM & SIM \\
\hline 2. Distribuição aleatória & SIM & SIM & SIM & SIM \\
\hline $\begin{array}{l}\text { 3. Alocação secreta dos } \\
\text { sujeitos }\end{array}$ & NÃO & NÃO & NÃO & NÃO \\
\hline $\begin{array}{l}\text { 4. Semelhança inicial } \\
\text { entre os grupos }\end{array}$ & SIM & SIM & SIM & SIM \\
\hline $\begin{array}{l}\text { 5. "Cegamento" dos } \\
\text { sujeitos }\end{array}$ & NÃO & NÃO & NÃO & NÃO \\
\hline $\begin{array}{l}\text { 6. "Cegamento" dos } \\
\text { terapeutas }\end{array}$ & NÃO & NÃO & NÃO & NÃO \\
\hline $\begin{array}{l}\text { 7. "Cegamento" dos } \\
\text { avaliadores }\end{array}$ & NÃO & NÃO & NÃO & NÃO \\
\hline $\begin{array}{l}\text { 8. Acompanhamento } \\
\text { adequado }\end{array}$ & SIM & SIM & SIM & SIM \\
\hline $\begin{array}{l}\text { 9. Análise da intenção } \\
\text { de tratamento }\end{array}$ & SIM & SIM & SIM & SIM \\
\hline $\begin{array}{l}\text { 10. Comparação } \\
\text { intergrupos }\end{array}$ & SIM & SIM & SIM & SIM \\
\hline $\begin{array}{l}\text { 11. Medidas de precisão e } \\
\text { variabilidade }\end{array}$ & SIM & SIM & SIM & SIM \\
\hline Escore Total & $07 / 10$ & $06 / 10$ & $06 / 10$ & 07/10 \\
\hline
\end{tabular}

Fonte: Pesquisa dos Autores.

Após análise e leitura, os estudos selecionados foram organizados em forma de tabela para apresentação do perfil das publicações. A Tabela 03 apresenta os estudos caracterizados de acordo com o ano de publicação, base de dados e idioma. O ano com concentrações iguais de estudos foi 2006, 2007, 2017 e 2019. Todos os estudos foram encontrados na base de dados PubMed, avaliados com a escala PEDro e estavam em idioma inglês. Esses dados evidenciam o maior contingente de publicações em âmbito internacional. 
Tabela 3 - Apresentação dos estudos de acordo com título, ano de publicação, autores, periódico, resultados. Teresina-PI, 2021.

\begin{tabular}{|l|l|}
\hline AUTORES & OBJETIVOS \\
\hline ANGIONE, et al., 2019 & Avaliar as alterações séricas em \\
& um grande painel de proteínas \\
& em pacientes com dor lombar \\
& crônica após tratamento em água \\
& aquecida.
\end{tabular}

METODOLOGIAS

Sessenta e seis pacientes com dor lombar crônica secundária à osteoartrite foram recrutados aleatoriamente e tratados com compressas diárias aquecidas e banhos de água mineral alcalina com bicarbonato, ou um esquema de reabilitação de hidroterapia térmica, a combinação dos dois regimes ou apenas medicação usual (grupo de controle), por duas semanas. As variáveis clínicas foram avaliadas no início do estudo, após 2 e 12 semanas.

ANDRADE, et al., 2017 Investigar $\mathrm{se} \quad \mathrm{o}$ treinamento físico aquático pode ajudar a melhorar a composição corporal e aumentar a capacidade funcional aeróbia em mulheres com sintomas de fibromialgia, e se o consumo de oxigênio (VO2) relacionado à massa corporal magra pode quantificar melhor a capacidade funcional dessa população.
Todas as mulheres foram submetidas ao teste cardiopulmonar de exercício (TCPE) para avaliar o consumo de oxigênio no limiar anaeróbio ventilatório (LAV) e no pico do exercício, e também para avaliar a composição corporal. $\mathrm{O}$ grupo treinado foi submetido ao programa de treinamento físico aquático (APT), realizado duas vezes por semana durante 16 semanas. A intensidade do exercício foi adaptada ao longo das sessões a fim de manter a frequência cardíaca e as classificações de esforço percebido alcançadas no VAT.
CARUS, et al., 2007
Avaliar os efeitos de um período de 12 semanas de treinamento aquático e subsequente sem tratamento na qualidade de vida relacionada à saúde (QVRS) e aptidão física em mulheres com fibromialgia.
Trinta e quatro mulheres com fibromialgia foram distribuídas aleatoriamente em dois grupos: um grupo de exercícios, que se exercitou por $60 \mathrm{~min}$ em água morna, três vezes por semana $(\mathrm{N}$ =17); e um grupo controle, que manteve suas atividades habituais de lazer $(\mathrm{N}=17)$. A QVRS foi avaliada por meio do questionário Short Form 36 e do Fibromyalgia Impact Questionnaire. A aptidão física foi medida usando os seguintes testes: Canadian Aerobic Fitness, dinamometria de preensão manual, caminhada de

\begin{abstract}
RESULTADOS
Todos os grupos de tratamento em água aquecida mostraram benefícios clínicos, conforme determinado por melhorias na dor, A terapia em água aquecida foi confirmada como benéfica para a dor crônica nas costas e provou induzir alterações nas proteínas envolvidas em funções como a modulação da expressão gênica.
\end{abstract}

Após o treinamento físico aquático, a composição corporal não foi significativamente diferente entre os grupos (GT e GC). No limiar anaeróbio ventilatório, apenas o TG apresentou aumento do VO2 relacionado à massa corporal magra, já que no TCP de pico, o $\mathrm{VO} 2$ em unidades absolutas, o $\mathrm{VO} 2$ relacionado à massa corporal total (TBM), o $\mathrm{VO} 2$ relacionado à massa corporal magra e a potência apresentaram diferenças significativas. As diferenças significativas entre o $\mathrm{VO} 2$ relacionado a massa corporal total e o $\mathrm{VO} 2$ relacionado à massa corporal magra basal e após 16 semanas de acompanhamento, tanto no TAV quanto no pico do TCPE em ambos os grupos. Foi observada também a diferença significativa entre o $\mathrm{VO} 2$ relacionado a massa corporal total e o $\mathrm{VO} 2$ relacionado a massa corporal total no VAT e no pico do teste cardiopulmonar de exercícios em ambos os grupos no início do estudo e após 16 semanas de acompanhamento.

Após 12 semanas de exercício aquático, efeitos positivos significativos do treinamento aquático foram encontrados na função física, dor corporal, percepção geral de saúde, vitalidade, função social, problemas emocionais e de saúde mental, equilíbrio e subir escadas. Após o período sem tratamento, apenas as melhorias na dor corporal e nos problemas emocionais do papel foram mantidas. 
$10 \mathrm{~m}$, subir escadas de 10 passos

e postura cega de uma perna. Os

resultados foram medidos no

início do estudo, após o

tratamento e após 3 meses sem

tratamento.

\begin{tabular}{ll}
\hline GUSI, et al., 2006 & Avaliar a eficácia de curto e \\
& longo prazo da terapia com \\
& exercícios em uma piscina \\
& aquecida na altura da cintura em \\
& mulheres com fibromialgia.
\end{tabular}

Trinta e quatro mulheres (média

+/- SD pontos dolorosos $17+/$ -

1) foram aleatoriamente

designados para um grupo de exercícios $(n=17)$ para realizar

3 sessões semanais de

treinamento, incluindo

exercícios aeróbicos,

$\begin{array}{lll}\text { proprioceptivos } & \mathrm{e} & \mathrm{de} \\ \text { fortalecimento } & \text { durante } & 12\end{array}$

A força dos extensores do joelho em ações concêntricas aumentou em $20 \%$ em ambos os membros após o período de treinamento, e essas melhorias foram mantidas após o período sem tratamento no grupo de exercício. A força de não mudou. A QVRS melhorou 12 em 93\% ( $\mathrm{P}=0,007)$ e a dor foi semanas, ou para um grupo de reduzida em $29 \%(\mathrm{P}=0,012)$ no controle $(\mathrm{n}=17)$. A força grupo de exercícios durante $\mathrm{o}$ isocinética unilateral máxima foi treinamento, mas a dor voltou medida nos extensores e perto do nível de pré-treinamento flexores do joelho em ações durante um período sem o concêntricas e excêntricas a 60 tratamento subsequente. No graus / segundo e 210 graus / entanto, não houve mudanças no segundo, e nos abdutores e grupo controle durante todo o adutores do ombro em período.

contrações concêntricas. A

qualidade de vida relacionada à saúde (QVRS) foi avaliada por meio do questionário EQ-5D; a dor foi avaliada em uma escala visual analógica. Todos foram medidos no início do estudo, pós-tratamento e após 6 meses.

Fonte: Pesquisa dos autores.

\section{Conclusão}

Este artigo de revisão expõe que os estudos apontam como a dor quando tratada na água aquecida tem uma melhora bastante significativa na qualidade de vida desses indivíduos, na capacidade física funcional, na fadiga e dentre outras. Para (Fioravanti, et al., 2011), os efeitos surgem mediados pelas propriedades físicas e químicas da água por meio de efeitos mecânicos e térmicos juntamente com o que se acredita contribuírem para os efeitos benéficos na via inflamatória e no metabolismo ósseo.

De acordo com (Angioni, et al., 2019), as doenças da coluna vertebral e as lombalgias crônicas são as afecções musculoesqueléticas mais prevalentes que afetam, ao longo da vida, a grande maioria da população. A dor nas costas é considerada crônica quando dura mais de dois/três meses. A água aquecida representa um tratamento popular para várias doenças reumáticas, como osteoartrite axial e periférica, fibromialgia e reumatismos extra articulares, mas também para artrite crônica como terapia adjuvante, e artrite reumatoide. Acredita-se que os efeitos terapêuticos benéficos dos tratamentos em água aquecida se devam principalmente às características físicas/químicas intrínsecas da água.

Para (Andrade, et al., 2017), estudos envolvendo treinamento físico aeróbio aquático em intensidades moderadas controladas pela Escala de Percepção de Esforço de Borg ou pelo método de reserva de frequência cardíaca têm apresentado resultados mais satisfatórios, entretanto, não há relatos na presente literatura de pesquisa sobre treinamento envolvendo exercícios físicos aeróbios no meio aquático com base na resposta alcançada no limiar anaeróbio ventilatório (VAT) no teste de exercício cardiopulmonar (CPET). Após a realização da pesquisa envolvendo 54 mulheres com fibromialgia houve efeitos positivos na capacidade funcional aeróbia durante o pico do teste de exercício cardiopulmonar, foi relatado também, que o 
treinamento físico aquático possui inúmeras vantagens trazidas pelas propriedades físicas da água e temperatura média contribuem efetivamente para aumentar a capacidade funcional aeróbia.

Segundo (Croisier, et al., 2002), os pacientes que apresentam dores musculares, fraqueza e fadiga podem eventualmente levar à redução da atividade física e diminuição da qualidade de vida. De acordo com (Altan, et al., 2006) e (Cedraschi, et al., 2004), algumas das terapias físicas experimentais para abordaram os efeitos do exercício em água morna, tentando combinar os efeitos revitalizantes e fortalecedores do exercício físico com as propriedades de relaxamento muscular do banho em água aquecida. Essas terapias têm se mostrado eficazes na redução dos sintomas e na melhoria da qualidade de vida relacionada à saúde (QVRS), o que normalmente é avaliado com o Fibromyalgia Impact Questionnaire (FIQ) ou o Short Form 36 (SF-36).

De acordo com (Carus, et al., 2007), após o estudo conclui que 3 meses de exercícios aquáticos melhoraram a qualidade de vida relacionada a à saúde, com grande alívio da dor e dos problemas emocionais, e melhorando a capacidade funcional física e o equilíbrio; as melhorias no condicionamento físico não duraram 12 semanas após o término do período de treinamento, enquanto alguns dos componentes da QVRS (e, particularmente, o alívio da dor e problemas emocionais) foram mantidos; e a alta taxa de adesão e adesão pode ser parcialmente atribuída ao desenho dos exercícios físicos (atividade física com música, jogos, etc.), que promoveu a interação entre os pacientes, e a interação com o monitor de exercícios.

Já (Gusi, et al., 2006) falam que, a força muscular depende tanto da área de seção transversal do músculo quanto da atividade neural, os tecidos musculares dos indivíduos que apresentam fibromialgia são caracterizados por anormalidades ultra estruturais e fragmentação de DNA não relacionadas à apoptose, envelhecimento ou descondicionamento. Nas piscinas aquecidas e exercícios terrestres mostraram uma capacidade semelhante de reduzir a dor e a fadiga, no entanto o impacto do exercício sobre a força é desconhecido e precisa de estudos mais detalhados. Eles também mostraram melhorar a capacidade cardiovascular, o tempo de caminhada e a saúde mental.

\section{Referências}

Almeida, D. C., \& Kraychete, D. C. (2017). Low back pain- A diagnostic approach. Pain Magazine, 16, 173-177.

Altan, L., Bingol, U., Aslan, M., \& Yurtkuran, M. (2006). The effect of balneotherapy on patients with ankylosing spondylitis. Scand. J. Rheumatol, 35, 283289.

Amorim, M. S., Saltiél, R. V; \& Sinhorim, L. (2018). Aquatic therapy in the treatment of low back pain: Literature Review. Movement\&Health •MAGAZINE INSPIRE. 48 (18).

Andrade, C. P., Zamunér, A. R., Forti, M., França, T. F., Tamburús, N. Y., \& Silva, E. (2017). Oxygen uptake and body composition after aquatic physical training in women with fibromyalgia: a randomized controlled trial. Eur J Phys Rehabil Med, 53 (5), $751-758$.

Angioni, M. M., Denotti, A., Sanna, C., Montisci, F., Dessole, G., Loi, A., \& Cauli, A. (2019). Spa therapy induces clinical improvement and protein changes in patients with chronic back pain. Rheumatism, 71 (3), 119-131.

Carus, P. T., Häkkinen., Arja., G N., Leal, A., Häkkinen, K., \& Alonso, A O. (2007). Aquatic training and detraining on fitness and quality of life in fibromyalgia. Med Sic Sport Exerc, 39 (7), 1044-50.

Cedraschi, C., Desmeules., Rapiti, E., Baumgartner, E., Cohen, P., Finckh, A., Allaz, A. F., \& Vischer, T. L. (2004). Fibromyalgia: a randomised, controlled trial of a treatment programme based on self management. Ann Rheum Dis, 63, 290-296.

Croisier, J. L., Renarde, C., \& Crielaard, J. M. (2002). Muscle performance in patients with fibromyalgia. Bone column joint, 69, $293-299$.

Fioravanti, A., Cantarini, L., Guidelli, G., \& Galeazzi, M. (2011). Mechanisms of action of thermal therapies in rheumatic diseases: What is the scientific evidence? Rheumatol Int, 31, 1-8.

Forazini, L. P. (2012). Health Sciences: Aquatic Physiotherapy. Paraná, Respiratório Unicentro: Ciência da saúde.

Guerreiro, V. M. (2016). Approach to adult low back pain in primary health care: review article. http://hdl.handle.net/10316/36798.

Gusi, N., Tomas, C P., Hakkinen, K A., Hakkinen, E A., \& Ortega, U. (2006). Exercise in warm water at waist level decreases pain and improves healthrelated quality of life and lower extremity strength in women with fibromyalgia. Artrite Rheum; 55 (1), 66-73.

Jentoft, E S., Kvalvik, A G., \& Mengshoel, A M. (2001). Effects of aerobic exercise in the pool and on land in women with chronic generalized fibromyalgia/muscle pain. Arthritis Rheum, 45, 42-47. 
Research, Society and Development, v. 10, n. 14, e183101422215, 2021

(CC BY 4.0) | ISSN 2525-3409 | DOI: http://dx.doi.org/10.33448/rsd-v10i14.22215

Larmer, P J., Bell, J., O'Brien, D., Dangen, J., \& Kersten, P. (2014). Hydrotherapy outcome measures for people with arthritis: a systematic review. NewZealand Journalof Physiotherapy, 2, 42.

Lisbôa, A A., Júnior, A C S., Lima, T B., \& De Almeida, R D. (2012). Effectiveness of aquatic physical therapy in the treatment of chronic low back pain: systematic review with meta-analyses. Graduate Notebook - Biological and Health Sciences, 1 (1), 33-42.

Milhões, A. K.., D, J. L., Croisier, C., Renarde, J. M., \& Crielaard. (2000). Pool exercises combined with an education program for patients with fibromyalgia syndrome, a prospective randomized trial. J Rheumatol, 27, 2473-2481.

Monteiro, S M R C. (2013). Changes in spinal curvature: Influence of Physiotherapy at the neuromuscular level. http://hdl.handle.net/10400.21/2915.

Nascimento, P. R. C., \& Costa, L. O. P. (2015). La prevalencia de dolor lumbaren Brasil: una revisión sistemática. Cad. Saúde Pública, 6, 31.

Sílvia, R. S., Costa, L. O. P., Moser, A. D. L., Aguiar, I. C., \& Oliveira, L. V. F. (2011). PEDro: thephysio therapy evidence databas. Physioter Mov. 24(3),523-533.

Sprott, H., Salemi, S., \& Gay, R. E. (2004). Increased DNA fragmentation and ultrastructural changes in fibromyalgia muscle fibers. Ann Rheum Dis, 63, 24551. 